\title{
The Study of Microbial Diversity in Mangrove Wetland Ecological System Based on a 16S rDNA Library
}

\author{
Huijie Liu ${ }^{1, *}$, Yun Tian ${ }^{2}$ \\ ${ }^{1}$ Guangzhou Military Area Environmental Monitoring Station, Guangzhou, China \\ ${ }^{2}$ State Key Laboratory of Marine Environmental Science, Xiamen University, Xiamen, China
}

Email address:

liuhja@126.com (Huijie Liu), tianyun@xmu.edu.cn (Yun Tian)

${ }^{*}$ Corresponding author

\section{To cite this article:}

Huijie Liu, Yun Tian. The Study of Microbial Diversity in Mangrove Wetland Ecological System Based on a 16S rDNA Library. International Journal of Environmental Monitoring and Analysis. Vol. 7, No. 2, 2019, pp. 48-55. doi: 10.11648/j.ijema.20190702.12

Received: January 3, 2019; Accepted: January 31, 2019; Published: September 10, 2019

\begin{abstract}
The microbial resources in mangrove wetland ecological system are abundant because of their special characteristics. However traditional methods of isolation and culture alone cannot analyze the microbial diversity fully and so, in this study, a 16S rDNA library was constructed to analyze microbial diversity in the Fugong mangrove of the Jiulong River Estuary, Fujian Province, China. The total sediment DNA was extracted, a 16S rDNA library constructed, and the clones analyzed using the restriction fragment length polymorphism (RFLP) method. The 16S rDNA sequences of 50 clones which had a higher display frequency in the RFLP analysis were blasted with the sequences in GenBank. The results showed that the highest similarity of the sequence in clones was $100 \%$, while the lowest was $88 \%$. The dominant microbes from mangrove sediments in the $16 \mathrm{~S}$ rDNA library belonged to the Proteobacteria $(70 \%)$ including $\alpha$-proteobacteria $(6.0 \%), \gamma$-proteobacteria $(22.0 \%)$, $\delta$-proteobacteria $(10.0 \%)$ and $\varepsilon$-proteobacteria $(32.0 \%)$. The other microbes were Bacteroidetes $(8.0 \%)$, Planctomycetacia (2.0\%), Actinobacteria (2.0\%) and Verrucomicrobia (2.0\%). Additional uncultured microorganisms as well as those whose classification information was unclear were also detected $(16.0 \%)$. The results of this study indicated that more objective and comprehensive information of microbial diversity in mangrove wetland ecological system had been obtained. There is abundant microbial diversity and a large amount of unknown microbial resources in mangrove wetland ecological system, which could have a very important potential, and so there should be more research to explore and utilize these microbial and functional gene resources in mangrove wetland ecological system.
\end{abstract}

Keywords: Mangrove Wetland, Ecological System, Microbial Diversity, 16S rDNA Library, Microbial Resources, Gene Resource

\section{Introduction}

The mangrove forest is a woody community that can be periodically submerged in seawater in the intertidal zone of tropical and subtropical regions [1]. The mangrove ecological system is made up of mangrove communities and their ambient environment. The structure and function of this ecological system combines marine and terrestrial properties but also has its own unique features: (1) the soil has deoxidized characteristics; (2) there is a higher salinity and salinization trend; (3) a lower $\mathrm{pH}$ value and higher acidity; and (4) a higher content of organic matter [2]. These systems are critical areas where biodiversity and valuable biological resources are very rich [3]. Because of their abundant biodiversity, these unique ecosystems have received attention from many scientists.

Microbial diversity is one important aspect of biodiversity, and because of their adaptation to the saline inter-tidal environment, bacteria and fungi are important in mangrove sediments and account for about 91\% [4]. Diverse types of bacteria have a certain similarity in different mangrove areas and the total microbial community play an important role in the carbon cycle and nitrogen fixation. Thus, knowledge of bacterial diversity in mangrove sediments is important for understanding how mangrove ecosystems function.

Since the 1940s, microbial diversity has been studied using the purified culture and isolation method. However, a number of microbes are often "viable but non-culturable". The 
quantity of microbial species, which have been cultured, isolated and described in the laboratory, is estimated to be only about $1 \%-5 \%$, and $95 \%-99 \%$ have not been isolated [5]. Thus, a little information has been revealed using these traditional methods, we do not have a full understanding of microbial diversity, since many of the microbial resources in the mangrove sediment have been ignored [6].

The increase in modern molecular biology technology has given the study of microbial biodiversity a new perspective [7, 8]. Using ribosomal RNA gene sequences as the criterion for identification, numerous new members belonging to known or new lineages of the bacteria and the archaeal domain have been detected [9]. A few studies have collectively provided useful insights into the phylogenetic composition of the prokaryotic community in coastal marine sediments. However, there are limited reports concerning microbial diversity in mangrove habitats, which have large ecological value. In this paper, microbial diversity has been studied by constructing a $16 \mathrm{~S}$ rDNA library, with some of the $16 \mathrm{~S}$ rDNA relating to genera or taxa that were classically identified in the Fugong mangrove of the Jiulong River Estuary, Fujian Province, China. The results showed that many were isolates from unknown genera, and thus extends our knowledge of microbial diversity in this unique coastal ecosystem.

\section{Materials and Methods}

\subsection{Study Sites and Sampling}

Fugong mangrove (Figure 1) is located to the south of the Jiulong River Estuary, and is a mangrove nature conservation area of Fugong, Fujian Province, China. The area of the mangrove community is about $670 \mathrm{~km}^{2}$, and Kandelia candel is the dominant mangrove species, with a little Aegiceras corniculatum distributed at the edges of the $K$. candel community [10]. On account of its geographical location, it has been affected by ship wastes, and the discharge of industrial, livestock, mariculture and household waste and wastewater.

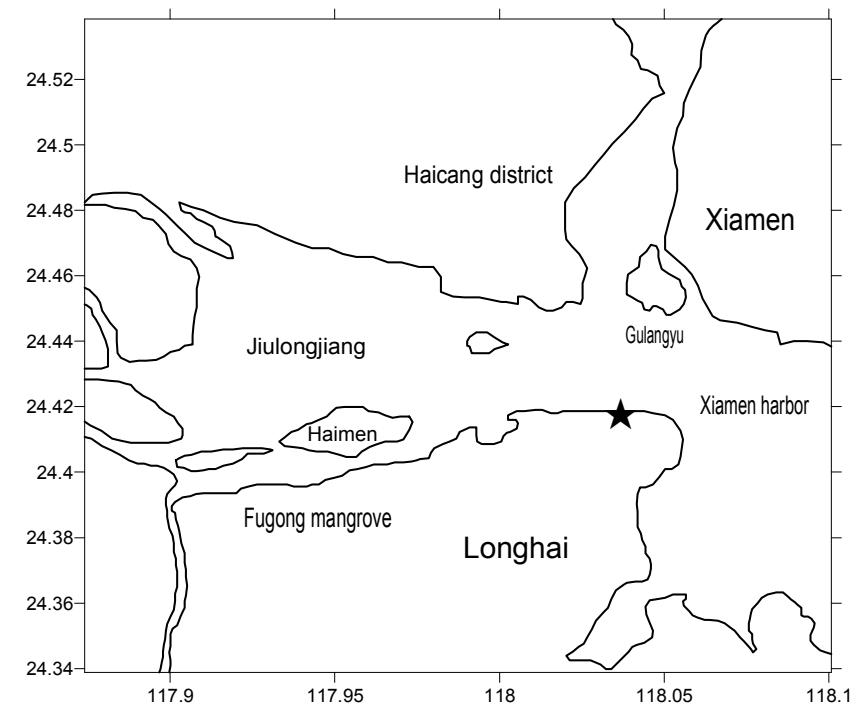

Figure 1. Map of Fugong mangrove area. The sampling site is indicated by an asterisk.
Three surface sediment samples $(0-5 \mathrm{~cm})$ including the low, medium and high tide zone were collected during low tides using a soil corer after the top centimeter of sediment was scraped off. A $500 \mathrm{~g}$ sediment sample was collected in each tidal zone, and these were then mixed together as a composite sample. Sediment samples were packed on-site into sealed polythene bags, and transported to the laboratory in an ice box for microbiological analysis within $24 \mathrm{~h}$ of collection [11].

\subsection{Extraction and Purification of the Sediment Genome DNA}

A weighed quantity ( $5 \mathrm{~g}$ ) of sediment was added to 13.5 $\mathrm{mL}$ soil DNA extraction buffer, and also $2 \%$ (final concentration) 2 - mercaptoethanol, and $5 \mathrm{mg} / \mathrm{mL}$ lysozyme were added. These were then mixed intensely, and placed in a $37^{\circ} \mathrm{C}$ water bath for 1 hour. Next, $2 \%$ SDS was added and they were placed in a $65{ }^{\circ} \mathrm{C}$ water bath for 2 hours, during which time they were mixed gently every 15 minutes. They were then centrifugated at $8500 \mathrm{~g}$ for 15 minutes and phenol chloroform solution $(1: 1, \mathrm{v} / \mathrm{v})$ and chloroform were added to the upper aqueous phase to remove the impurities. 0.25 volumes of ammonium acetate $(10 \mathrm{~mol} / \mathrm{L})$ and 0.6 volume of isopropanol were then added, and the mixture precipitated at room temperature for 1 hour and centrifugated ( $8500 \mathrm{~g}$ for $20 \mathrm{~min}$ ). The deposit was washed twice with $75 \%$ ethanol, and after the ethanol was discarded or volatilized, it was dissolved in $200-1000 \mu \mathrm{L}$ TE medium and the DNA was preserved at $-20^{\circ} \mathrm{C}$. The purification was performed using a GeneClean Turbo Kit from the Qbiogene Company.

\subsection{PCR Amplification and $16 S$ rDNA Gene Library Construction}

The bacterial 16S rDNA genes were amplified from the purified genome DNA of the mangrove sediment using the combination universal primers 27F (AGA GTT TGA TCC TGG CTC AG) and 1492R (GGT TAC CTT GTT ACG ACT T). The PCR reaction was performed using a thermal program, which comprised $94{ }^{\circ} \mathrm{C}$ for $5 \mathrm{~min}, 94^{\circ} \mathrm{C}$ for $1 \mathrm{~min}$, $55^{\circ} \mathrm{C}$ for $1 \mathrm{~min}, 72^{\circ} \mathrm{C}$ for $2 \mathrm{~min}, 30$ cycles; then $72^{\circ} \mathrm{C}$ for 10 min; then storage at $4^{\circ} \mathrm{C}$. The product was electrophoresed using $1 \%$ agarose gel and purified using the GeneClean Turbo Kit of the Qbiogene Company and then ligated by the pMD18-T vector, and transferred to Escherichia coli DH5 $\alpha$ competent cells. After blue/white screening, the positive clones were plated with ampicillin to construct the $16 \mathrm{~S}$ rDNA gene library.

\subsection{Analysis of the 16S rDNA Library with PCR-RFLP and Construction of a Phylogenetic Tree}

Primers M13-47 (5'-CGC CAG GGT TTT CCC AGT CAC GAC-3 ') and RV-M (5'-AGC GGA TAA CAA TTT CAC ACA GG-3') were selected to verify the size of the insert genes with the colony PCR in the original screened clones. Nested PCR was carried out with these verified positive clones as templates and 
the bacterial universal primers $27 \mathrm{~F}$ and $1492 \mathrm{R}$ as primers, and the resulting products were subjected to separate enzymatic digestions with $M s p$ I and Afa I endonuclease for restriction fragment length polymorphism (RFLP) analysis. The clones which had a higher frequency spectrum in the RFLP analysis were sequenced by the Yingjun Biotechnology Co., Ltd in Shanghai. The 16S rDNA sequences of these strains were aligned with the BLAST program on the NCBI website. The strain which had the highest similarity was used as a reference strain. If the similarity of the strains $\geq 97 \%$, they were regarded as the same genus [12]. Phylogenetic trees were built using DNAMAN software. One thousand bootstraps were performed to assign confidence levels to the nodes in the trees.

\section{Results}

\subsection{Total DNA Extraction of Mangrove Sediment}

The DNA size ranged from $2 \mathrm{~kb}$ to $23 \mathrm{~kb}$ according to the electrophoresis profile on agarose gels as a result of the existence of impurities such as humic acid. However, after being purified using the relevant protocols, the isolated DNA contained mainly
$23 \mathrm{~kb}$ molecules with a few smaller fragments, and its purity was also improved.

\section{2. $16 S$ rDNA Library Construction}

The fragment size of $1.5 \mathrm{~kb}$ was amplified in $16 \mathrm{~S}$ rDNA of purified samples using PCR in which the primers were Eubac $27 \mathrm{~F}$ and Eubac1492R. 500 white clones were randomly picked out to the LB plates with ampicillin after blue/white screening, then incubated at $37^{\circ} \mathrm{C}$ for 16 hours, before being stored at $4{ }^{\circ} \mathrm{C}$. The $16 \mathrm{~S}$ rDNA library of mangrove sediment was obtained.

\subsection{S rDNA Library Screening and RFLP Analysis}

The 250 DNA inserts from recombinant clones that were randomly selected in the 16S rDNA library were reamplified by PCR using the vector primers M13-47 and RV-M. The results of agarose gel electrophoresis indicated that most of the clones had been inserted into 16S rDNA fragments, as shown in Figure 2. Nested PCR was performed using the colony PCR product as template, and Eubac27F and Eubac1492R as primers. The 16S rDNA fragments with a DNA size of about $1.5 \mathrm{~kb}$ were amplified (Figure 3).

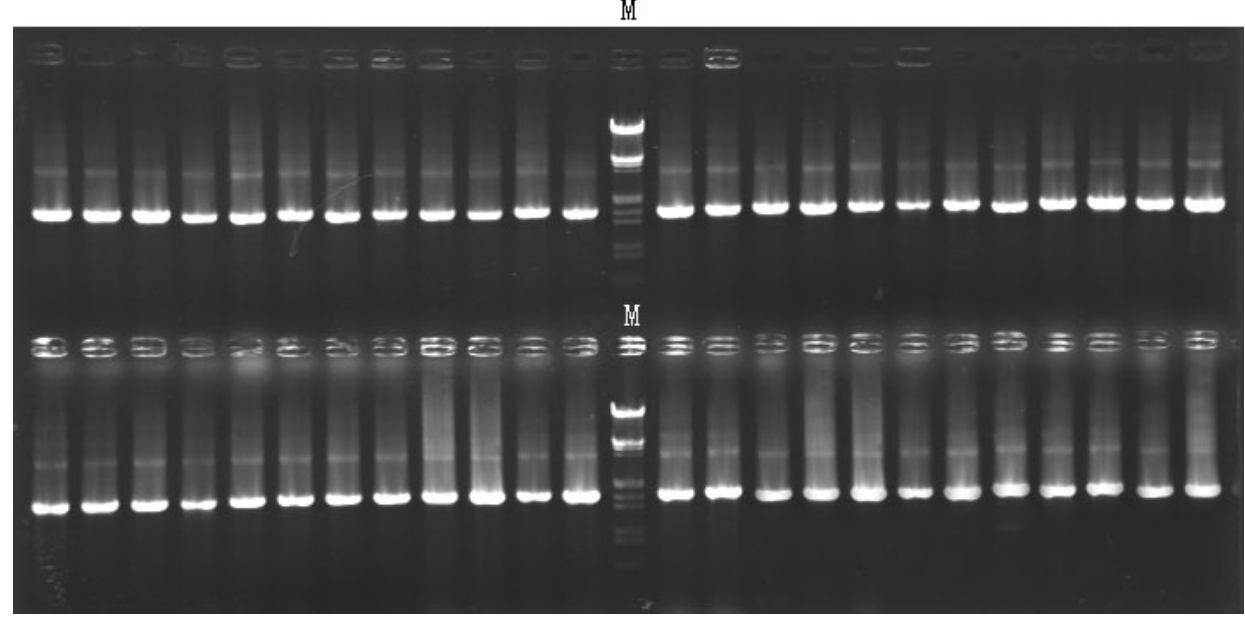

Figure 2. Partial results of colony PCR in 16 S rDNA library, M: Marker, SM0191.

M

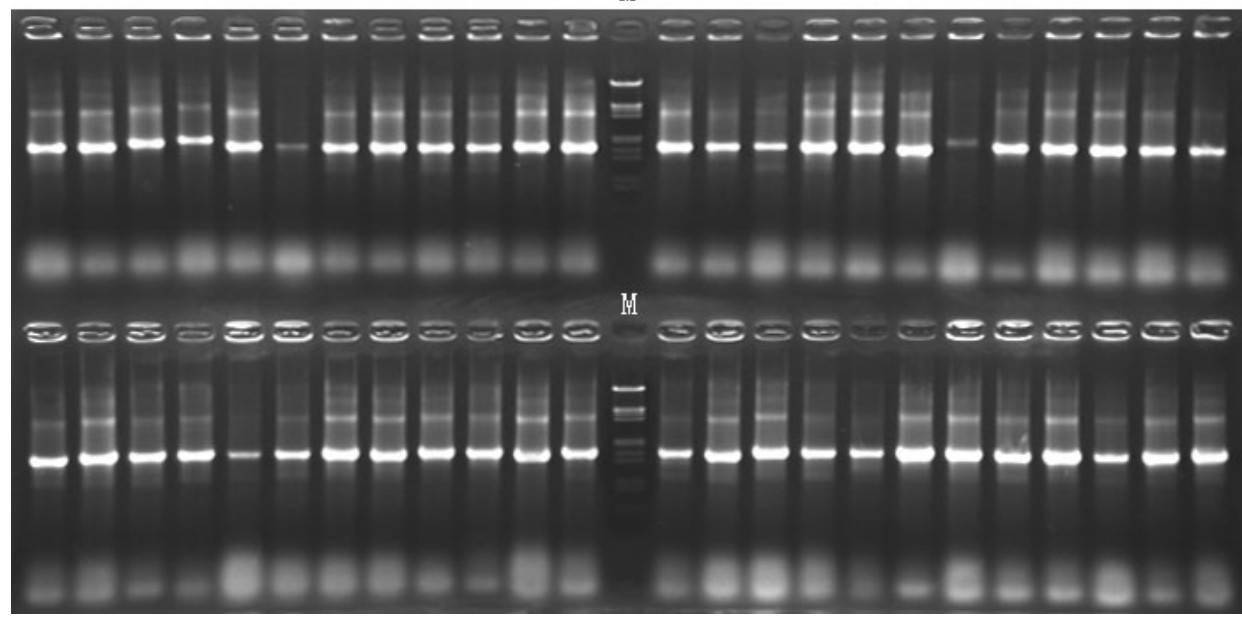

Figure 3. Partial results of nest PCR, M: Marker, SM0191. 
For RFLP analysis, the resulting products were subjected to separate enzymatic digestion Afa I (Takara) Msp I (Takara) endonucleases following the manufacturer's instructions, and the digested DNA fragments were run in 3\% agarose gels. After staining with ethidium bromide, the gels were photographed using an image-capture system equipped with a digital camera, and scanning image analyses were performed manually. The band patterns are shown in Figure 4. We analyzed the enzymic digestion profiles of 250 clones, resulting in the 50 clones with top frequency according to RFLP profiles being selected to determine sequence. These strains were considered as the predominant bacteria in mangrove sediments.

\section{M} $M$

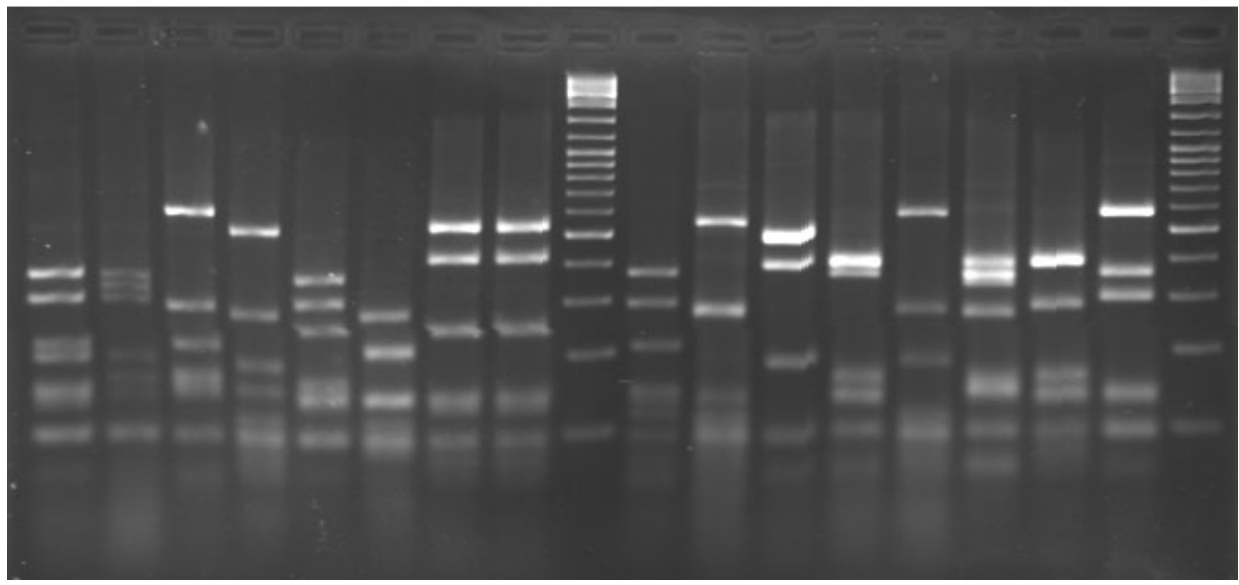

Figure 4. Partial results of RFLP analysis of positive colony in 16S rDNA library, M: Marker, SM0331.

\subsection{Phylogenetic Analysis of the Predominant Clones in the 16S rDNA Library}

The size of the $16 \mathrm{~S}$ rDNA clone fragments was about $1.5 \mathrm{~kb}$, and these sequences were blasted in GenBank after removal of the vector sequence by DNAstar software. The results are shown in Table 1, and the highest similarity to their closest counterparts in the public databases was $100 \%$ (the 25 th clone), while the lowest was $88 \%$ (the 42 th clone). The similarity of 35 clones in the $16 \mathrm{~S}$ library was no more than $97 \%$, accounting for $70 \%$. However, the similarity of 15 clones was more than $97 \%$ which accounted for $30 \%$. In addition, the most similar clone sequences in the library, compared with those in GenBank, were those of uncultured bacteria, such as Actinobacteria or Verrucomicrobia. The phylogenetic tree of 50 clones in the library is shown in Figure 5.

Various bacterial groups have been identified in mangrove sediment samples. The Proteobacteria group is predominant in the total sum, accounting for $70 \%$, including the $\alpha-, \gamma-, \delta$ - and $\varepsilon$-subgroups, accounting for $6.0 \%, 22.0 \%, 10.0 \%$ and $32.0 \%$, respectively. Moreover, Bacteroidetes, Planctomycetes, Actinobacteria and Verrucomicrobia were also analyzed, accounting for $8.0 \%, 2.0 \%, 2.0 \%$ and $2.0 \%$, respectively. Additionally, uncultured microorganisms and those whose classification information was unclear were also detected $(16.0 \%)$. Figure 6 shows the percentages of the different bacterial groups, based on the sum of the signals obtained from 50 clones in the library.

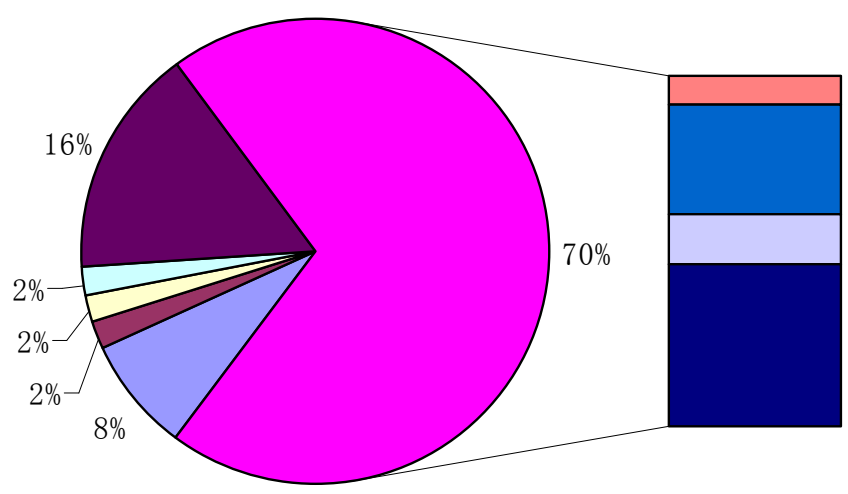

$6 \%$

$22 \%$

$10 \%$

$32 \%$

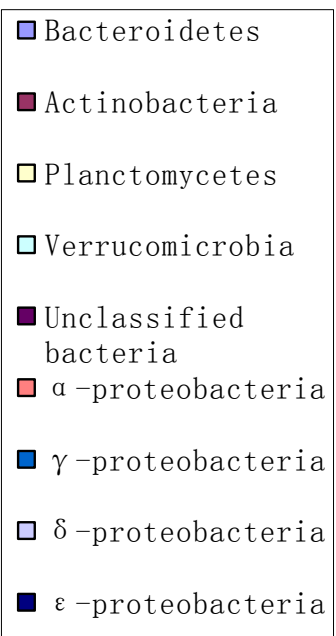

Figure 6. Classification and percentages of the 50 dominant bacteria froms mangrove sediment sample. 


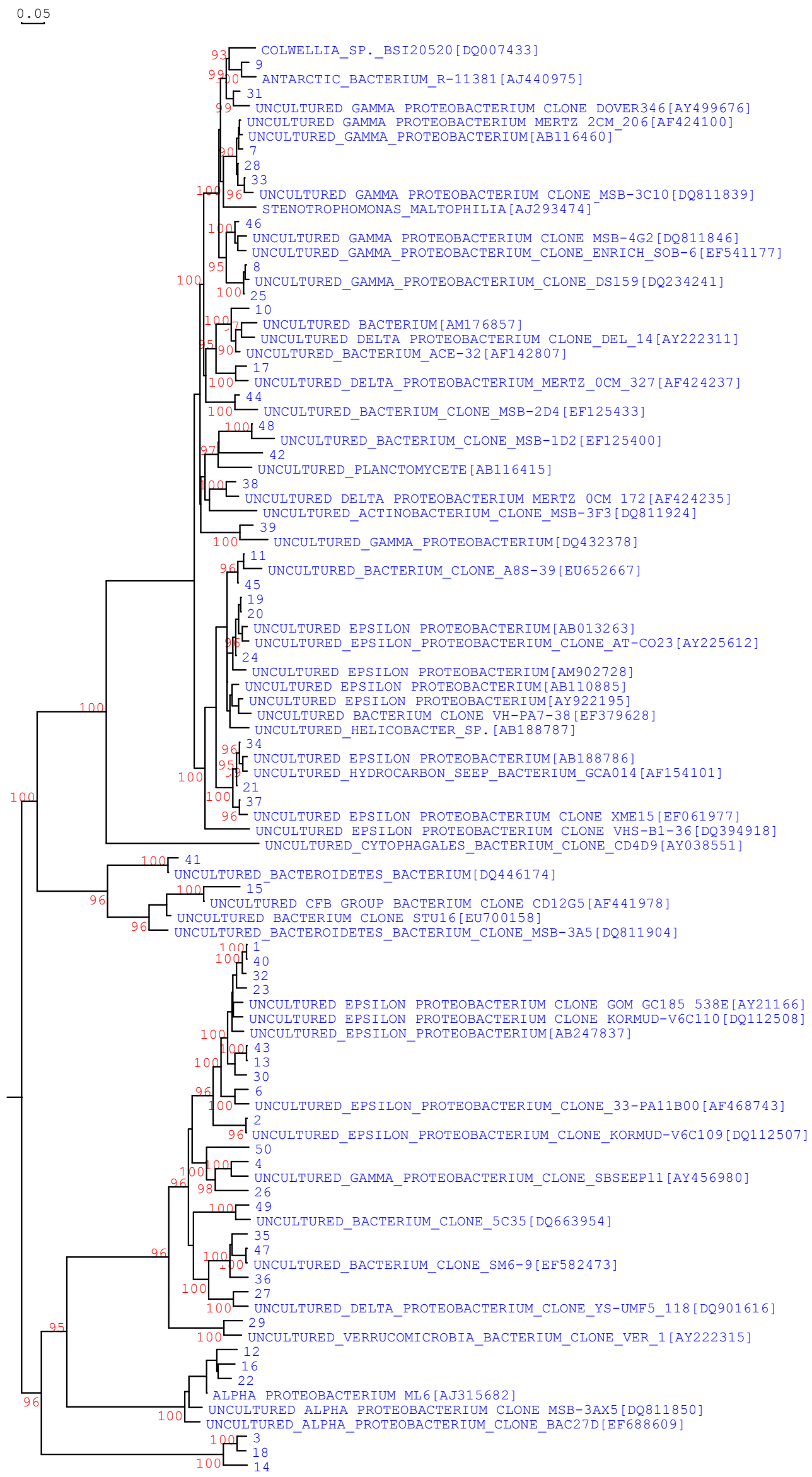

Figure 5. Phylogenetic tree of 50 dominant bacteria. 
Table 1. Analysis of bacterial diversity in Fugong mangrove sediments.

\begin{tabular}{|c|c|c|c|c|}
\hline Number & Most closely related sequence & Accession no. in GenBank & Similarity (\%) & Bacterial division \\
\hline 1 & Uncultured epsilon proteobacterium clone KorMud-V6C110 & DQ112508 & 95 & $\varepsilon$-proteobacteria \\
\hline 2 & Uncultured epsilon proteobacterium clone KorMud-V6C109 & DQ112507 & 99 & $\varepsilon$-proteobacteria \\
\hline 3 & Uncultured bacterium clone STU16 & EU700158 & 95 & Bacterium \\
\hline 4 & Uncultured gamma proteobacterium clone SBseep11 & AY456980 & 92 & $\gamma$-proteobacteria \\
\hline 5 & Uncultured actinobacterium clone MSB-3F3 & DQ811924 & 97 & Actinobacteria \\
\hline 6 & Uncultured epsilon proteobacterium clone & AF468743 & 93 & $\varepsilon$-proteobacteria \\
\hline 7 & Uncultured gamma proteobacterium MERTZ 2CM 206 & AF424100 & 99 & $\gamma$-proteobacteria \\
\hline 8 & Uncultured gamma proteobacterium clone enrich SOB-6 & EF541177 & 93 & $\varepsilon$-proteobacteria \\
\hline 9 & Antarctic bacterium R-11381 & AJ440975 & 97 & $\gamma$-proteobacteria \\
\hline 10 & Uncultured bacterium ACE-32 & AF142807 & 94 & $\delta$-proteobacteria \\
\hline 11 & Uncultured bacterium clone A8S-39 & EU652667 & 98 & Bacterium \\
\hline 12 & Uncultured alpha proteobacterium clone Bac27D & EF688609 & 98 & $\alpha$-proteobacteria \\
\hline 13 & Uncultured epsilon proteobacterium clone VHS-B1-36 & DQ394918 & 96 & $\varepsilon$-proteobacteria \\
\hline 14 & Uncultured Cytophagales bacterium clone CD4D9 & AY038551 & 97 & Bacteroidetes \\
\hline 15 & Uncultured CFB group bacterium clone CD12G5 & AF441978 & 92 & Bacteroidetes \\
\hline 16 & Uncultured alpha proteobacterium clone MSB-3ax5 & DQ811850 & 96 & $\alpha$-proteobacteria \\
\hline 17 & Uncultured delta proteobacterium MERTZ_0CM_327 & AF424237 & 95 & $\delta$-proteobacteria \\
\hline 18 & Uncultured Bacteroidetes bacterium clone $\overline{\mathrm{MSB}}-\overline{3} \mathrm{~A} 5$ & DQ811904 & 94 & Bacteroidetes \\
\hline 19 & Uncultured epsilon proteobacterium clone AT-co23 & AY225612 & 98 & $\varepsilon$-proteobacteria \\
\hline 20 & Uncultured epsilon proteobacterium & AM902728 & 96 & $\varepsilon$-proteobacteria \\
\hline 21 & Uncultured hydrocarbon seep bacterium GCA014, & AF154101 & 98 & $\varepsilon$-proteobacteria \\
\hline 22 & Alpha proteobacterium ML6 & AJ315682 & 96 & $\alpha$-proteobacteria \\
\hline 23 & Uncultured Helicobacter $\mathrm{sp}$. & AB188787 & 95 & $\varepsilon$-proteobacteria \\
\hline 24 & Uncultured epsilon proteobacterium clone: NKB11 & AB013263 & 97 & $\varepsilon$-proteobacteria \\
\hline 25 & Uncultured gamma proteobacterium clone DS159 & DQ234241 & 100 & $\gamma$-proteobacteria \\
\hline 26 & Stenotrophomonas maltophilia strain e-a22 & AJ293474 & 99 & $\gamma$-proteobacteria \\
\hline 27 & Uncultured delta proteobacterium clone YS-UMF5_118 & DQ901616 & 93 & $\delta$-proteobacteria \\
\hline 28 & Uncultured gamma proteobacterium clone: $\mathrm{Y} 2$ & $\mathrm{AB} 116460$ & 98 & $\gamma$-proteobacteria \\
\hline 29 & Uncultured Verrucomicrobia bacterium clone Ver 1 & AY222315 & 92 & Verrucomicrobia \\
\hline 30 & Uncultured bacterium clone VH-PA7-38 & EF379628 & 97 & Bacterium \\
\hline 31 & Uncultured gamma proteobacterium clone Dover 346 & AY499676 & 95 & $\gamma$-proteobacteria \\
\hline 32 & Uncultured epsilon proteobacterium clone: pLM10B-2 & AB247837 & 96 & $\varepsilon$-proteobacteria \\
\hline 33 & Uncultured gamma proteobacterium clone MSB-3C10 & DQ811839 & 98 & $\gamma$-proteobacteria \\
\hline 34 & Uncultured epsilon proteobacterium & $\mathrm{AB} 188786$ & 99 & $\varepsilon$-proteobacteria \\
\hline 35 & Uncultured delta proteobacterium clone Del 14 & AY222311 & 98 & $\delta$-proteobacteria \\
\hline 36 & Uncultured bacterium clone SZB57 & AM176857 & 96 & Bacterium \\
\hline 37 & Uncultured epsilon proteobacterium clone XME15 & EF061977 & 99 & $\varepsilon$-proteobacteria \\
\hline 38 & Uncultured delta proteobacterium MERTZ OCM 172 & $\mathrm{AF} 424235$ & 95 & $\delta$-proteobacteria \\
\hline 39 & Uncultured gamma proteobacterium clone $\overline{\mathrm{WN}}-\overline{\mathrm{HWB}}-160$ & DQ432378 & 90 & $\gamma$-proteobacteria \\
\hline 40 & Uncultured epsilon proteobacterium clone GoM GC185 538E & AY211663 & 95 & $\varepsilon$-proteobacteria \\
\hline 41 & Uncultured Bacteroidetes bacterium clone BBD_217_18 & DQ446174 & 99 & Bacteroidetes \\
\hline 42 & Uncultured planctomycete clone: KY128 & $\mathrm{AB} 116415$ & 88 & Planctomycetacia \\
\hline 43 & Uncultured epsilon proteobacterium clone 131625 & AY922195 & 97 & $\varepsilon$-proteobacteria \\
\hline 44 & Uncultured bacterium clone MSB-2D4 & EF125433 & 94 & Bacterium \\
\hline 45 & Uncultured epsilon proteobacterium clone: SEPR3279-63 & $\mathrm{AB} 110885$ & 95 & $\varepsilon$-proteobacteria \\
\hline 46 & Uncultured gamma proteobacterium clone MSB-4G2 & DQ811846 & 95 & $\gamma$-proteobacteria \\
\hline 47 & Uncultured bacterium clone Sm6-9 & EF582473 & 99 & Bacterium \\
\hline 48 & Uncultured bacterium clone MSB-1D2 & EF125400 & 95 & Bacterium \\
\hline 49 & Uncultured bacterium clone $5 \mathrm{C} 35$ & DQ663954 & 92 & Bacterium \\
\hline 50 & Colwellia sp. BSi20520 & DQ007433 & 95 & $\gamma$-proteobacteria \\
\hline
\end{tabular}

\section{Discussion}

There are special ecological environmental characteristics in mangrove ecosystems, which are important inter-tidal estuarine wetlands along the coastlines of tropical and subtropical regions. Thus, mangrove sediment provides abundant nutrients, carbon sources and growth factors for microorganisms [13], and their roles in mangrove habitats are well documented. In our previous studies, the total bacterial number in mangrove sediments is very high $\left(10^{9}\right.$ cell/g sediment) and most of them have a considerable potential for biodegradation of polycyclic aromatic hydrocarbons [14] and, moreover, the number of polycyclic aromatic hydrocarbon-degraders reaches $10^{4}-10^{5} \mathrm{cfu} / \mathrm{g}$ sediments [15]. Therefore, bacterial community diversity and persistence are important characteristics of mangrove habitat. Unfortunately, little information regarding the microbial communities in the Fugong mangrove habitat was obtained using traditional methods. However, during the last decade, molecular methods based on comparative analysis of $16 \mathrm{~S}$ rRNA sequences have yielded new and unexpected insights into the diversity of microbial communities. 16S rRNA sequences reveal numerous uncultured microorganisms in natural communities 
$[16,17]$. The $16 \mathrm{~S}$ rDNA library method which has been developed in recent years, overcame the deficiencies in the traditional methods of analysis of bacterial diversity, and reflects bacterial diversity more objectively and correctly. It is very important to realize the significance of microbial resources in mangrove sediments and explore them using the 16S rDNA library method.

In this study, we constructed a bacterial 16S rDNA library for mangrove sediment, and 250 clones from the library were selected for analysis with RFLP. Based on the top frequency from the RFLP results, 50 clones were considered as predominant strains with a high frequency. Comparing these clone sequences with those in the GenBank database, sequence similarity ranged from $88 \%$ to $100 \%$ to their closest counterparts in the public databases, and most of them $(92 \%)$ were uncultured microorganisms according to the alignment results. This implies that there are abundant uncultured microorganisms in mangrove sediments. Phylogenetic analysis revealed that the 50 bacterial clones fell into at least eight major lineages of the bacterial domain: the $\alpha-, \gamma-, \delta$-, $\varepsilon$-proteobacteria, Bacteroidetes, Planctomycetes, Actinobacteria, and Verrucomicrobia groups. The Proteobacteria group was predominant in the mangrove sediments and this agreed with a report that this group is the major type in most environments [18]. A total of 50 clones, represented by 35 sequence types and accounting for $70 \%$ of the gene library, grouped within the four subdivisions of the Proteobacteria. The $\varepsilon$-proteobacteria represented the most abundant proteobacterial group. Many of the sequence types were phylogenetically associated with cultivated organisms involved in S-cycles and most of the $\delta$-proteobacteria sequences clustered with members from genera with the well-known metabolic feature of anaerobic sulfate reduction, including Desulfotalea, Desulfobacterium, Desulfobulbus, and Syntrophobacter [19]. That $\delta$-proteobacteria have been detected would suggest that sulfate elements were abundant in the mangrove sediments. Bacteroides bacteria are closely associated with the conversion of organic matter, such as DNA, lipids and proteins, and their absorption and use by these bacteria is an important part of the carbon cycle in various environments [20]. Thus, it is rational to find them in mangrove sediments, which are rich in organic compounds. In general, low similarity is considered to indicate a new species in the GenBank database, and vice versa. A total of 46 clones, representing various sequence types and accounting for $92 \%$ of the gene library, were uncultured microorganisms. In addition, 36 sequence types displayed rather low levels of similarity to the rDNA gene sequences for cultivated and described members of the bacterial domain, and their exact phylogenetic affiliations remain unclear. It is very difficult to identify the physiological and biochemical characteristics and the ecological functions of the dominant microorganism in $s i t u$. If we want to know more concerning their structure and functioning, then more comprehensive, more particular and more intensive experiments need to be carried out in mangrove ecological systems.

\section{Conclusion}

Our results should lead to the consideration of new kinds of optimization strategies to study microbial diversity in the mangrove wetland ecological system. In this paper, microbial genetic information has been obtained on many uncultured forms for the mangrove wetland ecological system. Compared to the traditional method of bacterial isolation and culture, molecular identification techniques reflected information concerning the microbial community more objectively and comprehensively. Accordance to these results, it also indicated an abundant resource of uncultured microorganisms existing in the mangrove sediments. Their contribution to nature and man are incalculable and so, based on our study results, exploration and utilization of these special microbial resources may be of great significance in microbiology, ecology, bioremediation of environmental pollution, and so on.

\section{Acknowledgements}

This research was supported by the High-Tech Research and Development Program of China (No. 2008AA09Z408), NSF of China (No: 30530150, 40876061, 30800033), the Program for Changjiang Scholars and Innovative Research Team in University (No. 40821063) and the National Infrastructure of Natural Resources for Science and Technology Program of China (No. 2005DKA21209). We would like to thank Prof. I. J. Hodgkiss from The University of Hong Kong for his help with English.

\section{References}

[1] Pan Y, Chen J, Zhou H, et al. 2017. Vertical distribution of dehalogenating bacteria in mangrove sediment and their potential to remove polybrominated diphenyl ether contamination. Marine Pollution Bulletin, 124, 1055-1062.

[2] Priya G, Lau N S, Furusawa G, et al. 2019. Metagenomic insights into the phylogenetic and functional profiles of soil microbiome from a managed mangrove in Malaysia. Agri Gene, 9, 5-15.

[3] Hamzah T N T, Lee S Y, Hidayat A, et al. 2018. Diversity and characterization of endophytic fungi isolated from the tropical mangrove species, rhizophora mucronata, and identification of potential antagonists against the soil-borne fungus, fusarium solani. Frontiers in Microbiology, 9, 1707.

[4] Alongi D M. 1988. Bacterial productivity and microbial biomass in tropical mangrove sediments. Microbial Ecology, $15(1), 59-79$

[5] Rappe M S, Giovannoni S J. 2003. The uncultured microbial majority. Annual Review of Microbiology, 57, 369-94.

[6] Basak P, Pramanik A, Sengupta S, et al. 2016. Bacterial diversity assessment of pristine mangrove microbial community from Dhulibhashani, Sundarbans using 16S rRNA gene tag sequencing, Genomics Data, 7, 76-78.

[7] Rocha L L, Colares G B, Nogueira V L R, et al. 2016. Distinct Habitats Select Particular Bacterial Communities in Mangrove Sediments. International Journal of Microbiology, 2016, 1-6. 
[8] Handelsman J. 2004. Metagenomics: application of genomics to uncultured microorganisms. Microbiology and Molecular Biology Reviews, 68 (4), 669-85.

[9] Liu M, Yu Z, Yu X, et al. 2017. Invasion by cordgrass increases microbial diversity and alters community composition in a mangrove nature reserve. Frontiers in Microbiology, 8, 2503.

[10] Chen X Y, Lin P, Lin Y M. 1996. Mating systems and spontaneous mutation rates for chlorophyll-deficiency in populations of the mangrove Kandelia candel. Hereditas, 125 (1), 47-52.

[11] Liu H, Tian Y, Zheng T, et al. 2008. Studies of glucosidase activities from surface sediments in mangrove swamp. Journal of Experimental Marine Biology and Ecology, 367 (2), 111-117.

[12] Liang J B, Chen Y Q, Lan C Y, et al. 2007. Recovery of novel bacterial diversity from mangrove sediment. Marine Biology, 150 (5), 739-747.

[13] Li M, Gu J D. 2016. The diversity and distribution of anammox bacteria in the marine aquaculture zones. Appl Microbiol Biotechnol, 100, 8943-8953.

[14] Tian Y, Luo Y R, Zheng T L, et al. 2008. Contamination and potential biodegradation of polycyclic aromatic hydrocarbons in mangrove sediments of Xiamen, China. Marine Pollution Bulletin, 56 (6), 1184-1191.
[15] Tian Y, Liu H J, Zheng T L, et al. 2008. PAHs contamination and bacterial communities in mangrove surface sediments of the Jiulong River Estuary, China. Marine Pollution Bulletin, 57 (6-12), 707-15.

[16] Zhou Z, Meng H, Liu Y, et al. 2017. Stratified bacterial and archaeal community in mangrove and intertidal wetland mudflats revealed by high throughput 16S rRNA gene sequencing. Frontiers in Microbiology, 8, 2148.

[17] Ward D M, Weller R, Bateson M M. 1990. 16S rRNA sequences reveal numerous uncultured microorganisms in a natural community. Nature, 345, 63-65.

[18] Haldar S, Nazareth S W. 2018. Taxonomic diversity of bacteria from mangrove sediments of Goa: metagenomic and functional analysis. 3 Biotech, 8, 436.

[19] Paingankar M S, Deobagkar D D. 2018. Pollution and environmental stressors modulate the microbiome in estuarine mangroves: a metagenome analysis. Current Science, 115 (8), 1525-1535.

[20] Cottrell M T, Kirchman D L. 2000. Natural assemblages of marine proteobacteria and members of the Cytophaga-Flavobacter cluster consuming low- and high-molecular-weight dissolved organic matter. Applied and Environmental Microbiology, 66 (4), 1692-1697. 